\title{
REFERENCES
}

1. S. Aljančić, R. Bojanić, and M. Tomić, Sur la valeur asymptotique d'une classe des intégrales définies, Acad. Serbe Sci. Publ. Inst. Math. vol. 7 (1954) pp. 81-94.

2. E. B. Dynkin, Limit theorems for sums of independent random quantities, Izv. Akad. Nauk SSSR vol. 19 (1955) pp. 247-266 (Russian).

3. E. J. Gumbel, Statistics of extremes, New York, Columbia University Press, 1958.

4. J. Karamata, Sur une mode de croissance regulière des fonctions, Mathematica (Cluj) vol. 4 (1930) pp. 38-53.

5. S. Karlin and J. McGregor, Occupation time laws for birth and death processes, Technical Report No. 24, Contract Nonr-225(28), Stanford University, July 29, 1960.

6. J. Lamperti, Some limit theorems for stochastic processes, J. Math. Mech. vol. 7 (1958) pp. 433-450.

7. - An occupation time theorem for a class of stochastic processes, Trans. Amer. Math. Soc. vol. 88 (1958) pp. 380-387.

8. W. L. Smith, Asymptotic renewal theorems, Proc. Roy. Soc. Edinburgh. Sect. A vol. 64 (1954) pp. 9-48.

STANFORD UNIVERSITY

\section{ON THE INTERTWINING NUMBER THEOREM}

\section{ADAM KLEPPNER}

Let $G_{1}$ and $G_{2}$ be open subgroups of the separable locally compact group \& and let $L$ and $M$ be unitary representations of $G_{1}$ and $G_{2}$ respectively. For each $x$ and $y$ in $G$ the indices of the subgroup $H_{x, y}$ $=x^{-1} G_{1} x \cap y^{-1} G_{2} y$ in $x^{-1} G_{1} x$ and in $y^{-1} G_{2} y$ depend only on the $G_{1}: G_{2}$ double coset to which $x y^{-1}$ belongs. Let $\mathfrak{D}_{f}$ denote the set of all $G_{1}: G_{2}$ double cosets for which both these indices are finite and let $\mathfrak{D}$ denote the set of all $G_{1}: G_{2}$ double cosets. Let $\Im(L, M ; x, y)$ denote the intertwining number of the representations $s \rightarrow L_{x s x^{1}}=L_{s}^{x}$ and $s \rightarrow M_{y s y}{ }^{-1}$ $=M_{s}^{v}$ of $H_{x, y}$. The Intertwining Number Theorem of Mackey [1, Theorem $3^{\prime}$ ] states the following:

(1) $\Im(L, M ; x, y)$ depends only on the $G_{1}: G_{2}$ double coset $D$ to which $x y^{-1}$ belongs and may be denoted by $\mathfrak{S}(L, M ; D)$;

$$
\sum_{D \in D_{f}} \Im(L, M ; D) \leqq \Im\left(U^{L}, U^{M}\right) \leqq \sum_{D \in \mathscr{D}} \Im(L, M ; D) ;
$$

(3) if $L$ and $M$ are 1-dimensional then $\Im\left(U^{L}, U^{M}\right)=\sum_{D \in D_{f}} \Im(L, M ; D)$.

Received by the editors May 23, 1960. 
In his paper [1] Mackey raised the question of finding conditions under which the inequalities of (2) could be asserted to be equalities. It is the purpose of this note to show that if $L$ and $M$ are both finite dimensional then $\Im\left(U^{L}, U^{M}\right)=\sum_{D \in D_{f}} \Im(L, M ; D)$.

For each $y \in G$ and $w \in \mathfrak{E}(M)$ let $g_{y, w}$ be the function: $g_{y, w}(\eta y)$ $=M_{\eta}(w), \eta \in G_{2}$, and $g_{y, w}(z)=0, z \in G_{2} y$. Each $g_{y, w} \in \mathfrak{S}\left(U^{M}\right)$ and every function in $\mathfrak{S}\left(U^{M}\right)$ is a sum of functions $g_{y, w}$. Define analogously functions $f_{x, v} \in \mathfrak{S}\left(U^{L}\right)$ for each $x \in G$ and $v \in \mathfrak{G}(L)$. If $T$ is any intertwining operator for the representations $U^{L}$ and $U^{M}$ then for all $y \in \mathcal{B}$ and $w \in \mathfrak{S}(M)$

$$
T g_{y, w}=\sum_{\Theta / G_{1}}^{x} f_{x, A_{x, y}(w)},
$$

where $A_{x, y}: \mathfrak{S}(M) \rightarrow \mathfrak{S}(L)$ is a bounded linear operator and the sum is taken over any set of right coset representatives of $G_{1}$ in $\mathbb{G}$. Moreover

$$
T^{*} f_{x, v}=\sum_{\circledast / G_{2}}^{\nu} g_{y, A_{x, y}^{*}(v)}
$$

where $T^{*}$ and $A_{x, y}^{*}=\left(A_{x, y}\right)^{*}$ are the adjoint operators. The function $x, y \rightarrow A_{x, y}$ has the properties (a), (b), (c) of Lemma A of [1], namely:

$$
A_{\xi x, \eta y s}=L_{\xi} A_{x, y} M_{\eta}^{*}, \quad \text { for all } \xi \in G_{1}, \eta \in G_{2}, s \in \mathcal{B},
$$

(b) there exists a constant $K>0$ such that

$$
\sum_{\otimes / a_{1}}^{x}\left\|A_{x, y}(w)\right\|^{2} \leqq K\|w\|^{2}, \quad \text { for all } y \in \mathbb{S} \text { and } w \in \mathfrak{W}(M) \text {, }
$$

(c) there exists a constant $K^{\prime}>0$ such that

$$
\sum_{\Theta / G_{2}}^{y}\left\|A_{x, y}^{*}(v)\right\|^{2} \leqq K^{\prime}\|v\|^{2}, \quad \text { for all } x \in \mathfrak{S} \text { and } v \in \mathfrak{S}(L) .
$$

Because of (a) the value of $A$ at $x, y$ uniquely determines its values on the double coset $G_{1} x y^{-1} G_{2}$. Also by (a) the numbers $\left\|A_{x, y}(w)\right\|$ and $\left\|A_{x, y}^{*}(v)\right\|$ depend only on the $G_{1}$ coset to which $x$ belongs and the $G_{2}$ coset to which $y$ belongs so that the sums in (b) and (c) are well defined. Each $A_{x, y}$ is an intertwining operator for the representations $L^{x}$ and $M^{y}$ of $H_{x, y}[1$, Lemma D]. Conversely, let $A$ be an intertwining operator for $L^{x}$ and $M^{y}$. Set $A_{\xi x s, \eta y s}=L_{\xi} A M_{\eta}^{*}$ for $\xi \in G_{1}, \eta \in G_{2}$, $s \in \mathcal{B S}$, and $A_{x^{\prime}, y^{\prime}}=0$ for $x^{\prime} y^{\prime-1} \in G_{1} x y^{-1} G_{2}$. Defining an operator $T$ by $\left.{ }^{*}\right)$ yields a possibly unbounded intertwining operator for $U^{L}$ and $U^{M}$. It is this correspondence between operator-valued functions 
$A_{x, y}$ and intertwining operators $T$ which yields the intertwining number theorem. The problem of showing that

$$
\Im\left(U^{L}, U^{M}\right)=\sum_{D \in D_{f}} \Im(L, M ; D)
$$

reduces to showing that if $x, y \rightarrow A_{x, y}$ is defined by a bounded intertwining operator as in (*) and if $A_{x, y} \neq 0$ for some particular $x, y$ then the double coset $D=G_{1} x y^{-1} G_{2} \in \mathfrak{D}_{f}$.

Let $\left\{r_{j}\right\}$ be a set of right coset representatives of $H_{x, y}$ in $y^{-1} G_{2} y$. Then $G_{1} x y^{-1} G_{2}$ is a disjoint union of cosets $G_{1} x r_{j} y^{-1}$. It follows from (a) that $A_{x r_{j} y^{-1, e}}=A_{x, y r_{j}^{-1} y^{-1} y}=A_{x, y} M_{r_{j}}^{y}$ and from (b) that

$$
\sum_{j}\left\|A_{x, y} M_{r_{j}}^{y}(w)\right\|^{2} \leqq K\|w\|^{2}, \quad \text { all } w \in \mathfrak{S}(M) .
$$

Suppose that $M$ is finite dimensional and assume that $\left[y^{-1} G_{2} y: H_{x, y}\right]$ $=\infty$, i.e. that the set $\left\{r_{j}\right\}$ is infinite. Let $N$ be the null space of $A_{x, y}$ and let $w_{1}, \cdots, w_{m}$ be an orthonormal basis of $\mathfrak{S}(M)$. Because the unit ball in $\mathfrak{S}(M)$ is compact there exists a subsequence $\left\{r_{j_{k}}\right\}$ such that $M_{r_{j_{k}}}^{\nu}\left(w_{i}\right)$ converges to $w_{i}^{\prime}$ for each $i=1, \cdots, m . w_{1}^{\prime}, \cdots, w_{m}^{\prime}$ is also an orthonormal basis of $\mathfrak{W}(M)$ and because $A_{x, y} \neq 0$ there exists an index $i$ such that $w_{i}^{\prime} \notin N$. Hence $\left\|A_{x, y}\left(w_{i}^{\prime}\right)\right\|>c>0$. There exists $d>0$ such that if $\left\|w-w_{i}^{\prime}\right\|<d$ then $\left\|A_{x, y}(w)\right\|>c$. For $k$ sufficiently large $\left\|M_{r_{j_{k}}}^{\nu}\left(w_{i}\right)-w_{i}^{\prime}\right\|<d$; hence $\left\|A_{x, y} M_{r_{j_{k}}}^{\nu}\left(w_{i}\right)\right\|>c$ for $k$ sufficiently large. But this contradicts $(* * *)$ and the set $\left\{r_{j}\right\}$ must be finite. A similar argument applied to $A_{x, y}^{*}$ shows that $\left[x^{-1} G_{1} x: H_{x, y}\right]<\infty$ when $L$ is finite dimensional. Thus $D=G_{1} x y^{-1} G_{2} \in \mathfrak{D}_{f}$.

\section{REFERENCE}

1. G. W. Mackey, On induced representations of groups, Amer. J. Math. vol. 73 (1951) pp. 576-592.

\section{HARVARD UNIVERSITY}

